\title{
Integration von privater und gesetzlicher Krankenversicherung vor dem Hintergrund internationaler Erfahrungen
}

\author{
Stefan Greß \\ Simone Leiber \\ Maral Manouguian
}

Die in Deutschland bestehende Trennung zwischen privater und gesetzlicher Krankenversicherung ist in Europa inzwischen einmalig und ruft vielfältige Probleme hervor. Vor der Bundestagswahl 2005 herrschte in der Wissenschaft ein recht breiter Konsens, diese Trennung aufzuheben. Dies fand sowohl bei den Befürwortern einer einkommensabhängigen Bürgerversicherung Zustimmung als auch bei einigen Anhängern einkommensunabhängiger Pauschal- bzw. Bürgerprämien. Der Gesetzgeber schreckte allerdings vor grundlegenden Veränderungen zurück. Nun steht die Bundestagswahl 2009 bevor. Dieser Beitrag diskutiert vor dem Hintergrund internationaler Erfahrungen, welche Optionen dem Gesetzgeber zur Herstellung einer einheitlichen Wettbewerbsordnung zur Verfügung stehen.

\section{Einleitung}

Im deutschen Krankenversicherungssystem existieren gesetzliche Krankenversicherung (GKV) und private Krankenvollversicherung (PKV) nebeneinander. Mit Ausnahme der USA ist dieses duale System im internationalen Vergleich entwickelter Industrieländer einzigartig. Zuletzt haben im Jahr 2006 auch die Niederlande beide Krankenversicherungssysteme integriert. Dies geschah aus gutem Grund, schließlich bringt das Nebeneinander von sozialer Krankenversicherung und privater Krankenvollversicherung eine Reihe von gravierenden Problemen mit sich. Diese Probleme resultieren in Deutschland daraus, dass für beide Systeme unterschiedliche Rahmenbedingungen gelten, insbesondere bei der Kalkulation der Beiträge bzw. Prämien und der Vergütung der Leistungserbringer.

Dieser Beitrag analysiert zunächst die Funktion privater Krankenversicherung im internationalen Vergleich - auch vor dem Hintergrund der Schaffung einer einheitlichen Wettbewerbsordnung in den Niederlanden (Abschnitt 2). Im Folgenden wird diskutiert, welche Probleme sich aus dem Nebeneinander von privater Krankenvollversicherung und sozialer Krankenversicherung in Deutschland ergeben. Dabei werden auch die Auswirkungen der jüngsten Gesundheitsreform berücksichtigt (Abschnitt 3). Abschnitt 4 skizziert dann abschließend vor dem Hintergrund der internationalen Erfahrungen und der aus dem

\begin{tabular}{|c|c|c|c|c|}
\hline \multirow[t]{2}{*}{ Land } & \multirow{2}{*}{$\begin{array}{l}\text { Vorherrschende } \\
\text { Finanzierungsform }\end{array}$} & \multicolumn{3}{|c|}{ Art des privaten Krankenversicherungsschutzes } \\
\hline & & Substitutiv* & Komplementär** & Parallel*** \\
\hline Deutschland & Beiträge & + & + & - \\
\hline Niederlande & Beiträge & $+($ bis 2005) & + & - \\
\hline Frankreich & Beiträge & - & + & - \\
\hline Österreich & Beiträge & - & + & - \\
\hline USA & Privat & + & + & - \\
\hline Kanada & Steuern & - & + & + \\
\hline Großbritannien & Steuern & - & + & + \\
\hline
\end{tabular}

Nebeneinander von GKV und PKV resultierenden Probleme Optionen für die Integration beider Krankenversicherungssysteme.

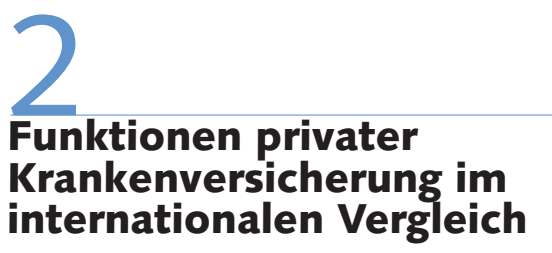

Die Funktion privater Krankenversicherungen wird nicht nur in Deutschland kontrovers diskutiert (Colombo/Tapay 2004; Mossialos/Thomson 2004). Die Schwerpunkte dieser Diskussion unterscheiden sich jedoch in Abhängigkeit von der Funktion, die privater Krankenversicherungsschutz im jeweiligen Gesundheitssystem erfüllt. Idealtypisch lassen sich drei Funktionen unterscheiden: substitutiver, komplementärer und paralleler Krankenversicherungsschutz (Tabelle 1).
In den entwickelten Industrieländern gibt es nur drei Länder, in denen es substitutiven privaten Krankenversicherungsschutz gibt bzw. bis vor Kurzem gegeben hat. Substitutiv bedeutet, dass ein Teil der

Stefan Greß, Prof. Dr., Professor für Versorgungsforschung und Gesundheitsökonomie an der Hochschule Fulda. Arbeitsschwerpunkte: Krankenversicherungsökonomie, Internationaler Gesundheitssystemvergleich, Versorgungsforschung. e-mail: stefan.gress@hs-fulda.de Simone Leiber, Dr., Wissenschaftlerin im WSI in der Hans-Böckler-Stiftung. Arbeitsschwerpunkte: Wohlfahrtsstaaten im Vergleich, Europäische Sozialpolitik, Gesundheitspolitik.

e-mail: Simone-Leiber@boeckler.de Maral Manouguian, Doktorandin im WSI in der Hans-Böckler-Stiftung. Arbeitsschwerpunkt: Gesundheitsökonomie. e-mail: Maral-Manouguian@boeckler.de 
Bevölkerung privaten Krankenversicherungsschutz als Vollversicherung wählen kann. In den USA betrifft dies nahezu die gesamte Bevölkerung unter 65 Jahren, da für diesen Personenkreis weder ein beitrags- noch ein steuerfinanziertes Gesundheitssystem zur Verfügung steht. Nur Bedürftige - insbesondere solche mit Kindern - haben unter Umständen Zugang $\mathrm{zu}$ einer steuerfinanzierten Grundversorgung. Knapp $20 \%$ der Bevölkerung unter 65 Jahren finden in den USA keinen $\mathrm{Zu}$ gang zu bezahlbarer Krankenvollversicherung (Carey et al. 2009).

In den Niederlanden mussten bis 2005 alle Erwerbstätigen mit einem Einkommen oberhalb einer Berechtigungsgrenze aus der sozialen Krankenversicherung ausscheiden und waren auf private Krankenvollversicherung angewiesen. Dies betraf etwa $30 \%$ der Bevölkerung. Durch die umfassende Regulierung der privaten Krankenvollversicherung lag der Anteil der Nichtversicherten in den Niederlanden im Jahr 2005 unter $1 \%$. Die 2006 implementierte Krankenversicherungsreform in den Niederlanden integrierte beide Krankenversicherungssysteme. Formalrechtlich führte die Reform zu einer Privatisierung der sozialen Krankenversicherer (Rosenau/Lako 2008). Die Unterscheidung zwischen privaten Versicherungsgesellschaften und vormaligen Krankenkassen verschwimmt allerdings aufgrund der ausgeprägten staatlichen Rahmenregulierung der privaten Krankenversicherungsgesellschaften (Leiber/Manouguian 2009). Und auch vor der Reform kooperierten private und öffentliche Krankenversicherer - anders als in Deutschland - bereits sehr eng. Insgesamt resultierte die Reform in der Einführung einer einheitlichen Wettbewerbsordnung ${ }^{1}$ unter Ausschluss risikobezogener Prämien und mit Kontrahierungszwang für alle Krankenversicherer. Festzuhalten ist auch, dass die formale Integration der beiden Krankenversicherungssysteme nur der letzte Schritt in einem langen Reformprozess war, der durch eine Reihe von Maßnahmen beide Versicherungssysteme aneinander angeglichen hat. $\mathrm{Zu}$ diesen Maßnahmen zählten insbesondere die Angleichung der Vergütungssysteme in der ambulanten ärztlichen Versorgung und die Einführung eines gesetzlich regulierten Basistarifs (Greß et al. 2007). Nach der Integration der beiden Krankenversicherungssysteme in den Niederlanden ist Deutschland neben den USA unter den westlichen Industrielän- dern inzwischen das einzige Land, in dem private Krankenversicherung eine substitutive Funktion erfüllt.

In allen entwickelten Industrieländern (einschließlich Deutschland und USA) nimmt private Krankenversicherung eine komplementäre Funktion ein, indem im öffentlichen System nicht oder nicht vollständig abgedeckte Leistungen versichert werden (private Krankenzusatzversicherung). Die Größe des entsprechenden Marktes ist abhängig vom Umfang des durch Beiträge oder Steuern versicherten Leistungskatalogs. Eine öffentliche Regulierung von $\mathrm{Zu}$ satzversicherungen ist zumindest in der Europäischen Union aufgrund entsprechender Regelungen im Gemeinschaftsrecht nicht möglich. Daraus ergeben sich Zugangsprobleme bzw. hohe finanzielle Belastungen für schlechte Risiken, wenn das öffentlich finanzierte Leistungspaket wie etwa in Frankreich - besonders große Lücken hat (Greß/Wasem 2003).

Die dritte Funktion privater Krankenversicherung besteht darin, durch die Entrichtung privater Krankenversicherungsprämien ein Anrecht auf bevorzugte Behandlung zu erwerben, obwohl parallel Beiträge oder Steuern gezahlt werden. Ein Markt für diesen parallelen Krankenversicherungsschutz besteht derzeit vor allem in vorwiegend steuerfinanzierten Gesundheitssystemen mit Kapazitätsproblemen, wie etwa in Großbritannien und Kanada. Kritisch diskutiert wird paralleler privater Krankenversicherungsschutz vor allem deshalb, weil diese Art von privater Krankenversicherung durch finanzielle Anreize für Leistungserbringer die bevorzugte Behandlung des privat versicherten Personenkreises sicherstellen soll. Bestehende Kapazitätsprobleme im öffentlich finanzierten System werden so verschärft. Aus diesem Grund verbieten etwa einige kanadische Provinzen das Angebot von parallelem privaten Krankenversicherungsschutz (Greß 2005, 2007).

\section{Probleme des Neben- einanders von GKV und PKV in Deutschland}

Wie oben beschrieben, hat in Deutschland private Krankenversicherung sowohl eine substitutive als auch eine komplementäre Funktion. Das Angebot privater Zusatz- versicherungen ist wegen des im internationalen Vergleich in Deutschland sehr umfassenden Leistungskatalogs relativ unproblematisch, auch wenn durch die im Rahmen des GKV-Modernisierungsgesetzes im Jahr 2004 eingeleitete Aufhebung der strikten Trennung zwischen gesetzlicher Krankenversicherung und privater Zusatzversicherung der Einsatz von $\mathrm{Zu}-$ satzversicherungen als Selektionsinstrument wahrscheinlicher wird.

Probleme ergeben sich im deutschen Krankenversicherungssystem vielmehr durch das Nebeneinander von sozialer Krankenversicherung und substitutivem privaten Krankenversicherungsschutz. Sie sind Konsequenz unterschiedlicher Regelungen bei der Kalkulation von Beiträgen bzw. Prämien und bei den Vergütungssystemen, insbesondere in der ambulanten ärztlichen Versorgung.

Die Beiträge in der GKV werden unabhängig vom gesundheitlichen Risiko und abhängig vom Einkommen erhoben (Übersicht 1). Kinder und nicht erwerbstätige Ehegatten werden beitragsfrei mitversichert. Die privaten Krankenversicherer kalkulieren dagegen Prämien auf der Grundlage des gesundheitlichen Risikos und unabhängig vom Einkommen. Kinder sowie nicht erwerbstätige Ehegatten müssen separat versichert werden und zahlen ebenfalls Prämien. Diese Unterschiede sind bedeutsam, weil ein Teil der in der GKV Versicherten in die PKV wechseln kann. Insbesondere Selbstständige und Versicherte mit einem Einkommen oberhalb der Versicherungspflichtgrenze haben diese Möglichkeit. Attraktiv ist der Wechsel in die PKV vor allem für gesunde Singles mit hohem Einkommen. Diese Personengruppe muss in der PKV weder Risikoaufschläge zahlen, noch muss sie Prämien für Familienangehörige finanzieren. Im Umkehrschluss ist für chronisch Kranke und Familien ein Wechsel in die private Krankenversicherung nicht attraktiv. Die vorhandene Empirie zeigt dann auch eindeutig, dass Versicherte in der PKV ein deutlich höheres Durchschnittseinkommen haben als jene in der GKV und im Durchschnitt deutlich gesünder sind (Mielck/Helmert

Darunter verstehen wir die Aufhebung des dualen Systems der PKV und GKV und Überführung der Krankenvollversicherung in einen einheitlichen Regulierungsrahmen für die Krankenversicherungsträger, unabhängig davon, ob sie privatrechtlich oder öffentlich-rechtlich organisiert sind. 


\begin{tabular}{|c|c|c|}
\hline \multicolumn{3}{|c|}{ Übersicht 1: Gesetzliche und private Krankenversicherung im Vergleich } \\
\hline & $\begin{array}{l}\text { Gesetzliche } \\
\text { Krankenversicherung } \\
\text { (ca. } 70 \text { Mio. Versicherte) }\end{array}$ & $\begin{array}{l}\text { Private Krankenversicherung } \\
\text { (ca. } 9 \text { Mio. Versicherte) }\end{array}$ \\
\hline Rechtsform & Öffentlich-rechtlich & $\begin{array}{l}\text { Privatrechtlich } \\
\text { (Versicherungsvereine auf } \\
\text { Gegenseitigkeit und } \\
\text { Kapitalgesellschaften) }\end{array}$ \\
\hline Finanzierung & Umlageverfahren & Kapitaldeckungsverfahren \\
\hline Einnahmebasis & $\begin{array}{l}\text { Einkommensabhängige Beiträge } \\
\text { und Zuschüsse des Bundes }\end{array}$ & Risikoäquivalente Prämien \\
\hline Kontrahierungszwang & ja & $\begin{array}{l}\text { Begrenzt (de facto für Beamte; } \\
\text { ab } 2009 \text { verpflichtend im Basistarif) }\end{array}$ \\
\hline Eintrittsbarrieren & $\begin{array}{l}\text { beschränktes Rückkehrrecht } \\
\text { nach PKV-Versicherung }\end{array}$ & $\begin{array}{l}\text { Einkommen muss drei Jahre über } \\
\text { der Versicherungspflichtgrenze } \\
\text { liegen oder Nachweis des Beamten- } \\
\text { tums bzw. einer selbstständigen } \\
\text { Tätigkeit }\end{array}$ \\
\hline $\begin{array}{l}\text { Beitragsfreie Mitversicherung } \\
\text { von Ehepartnern und Kindern }\end{array}$ & ja & nein \\
\hline Leistungsumfang & $\begin{array}{l}\text { Mindestleistungen werden } \\
\text { gesetzlich festgelegt und können } \\
\text { durch den Gesetzgeber erweitert } \\
\text { oder beschränkt werden }\end{array}$ & $\begin{array}{l}\text { Leistungen können je nach Tarif } \\
\text { vereinbart werden (Ausnahme } \\
\text { Basistarif) }\end{array}$ \\
\hline Risikostrukturausgleich & Morbiditätsorientierter RSA & $\begin{array}{l}\text { Ausgabenausgleich für Defizit } \\
\text { im Basistarif }\end{array}$ \\
\hline $\begin{array}{l}\text { Vergütung der } \\
\text { Leistungserbringer }\end{array}$ & $\begin{array}{l}\text { Fallpauschalen und Einzel- } \\
\text { leistungsvergütung bis zur Grenze } \\
\text { des Regelleistungsvolumens }\end{array}$ & $\begin{array}{l}\text { Einzelleistungsvergütung } \\
\text { ohne Mengenobergrenzen }\end{array}$ \\
\hline
\end{tabular}

2006a). Durch diese negative Auslese wird die Nachhaltigkeit der Finanzierung in der GKV geschwächt. Eine Ausweitung des GKV-Versichertenkreises um freiwillig privat Versicherte, Beamte und Selbstständige könnte zu einer - allerdings einmaligen Senkung der GKV-Beitragssätze beitragen. ${ }^{2}$ Der Finanzierungsmechanismus in der GKV beruht auf dem Umlageverfahren sowie der Einkommenssolidarität (Umverteilung von Personen mit hohem Einkommen zu Personen mit niedrigem Einkommen) und Risikosolidarität (Umverteilung von Gesunden zu Kranken). Die Funktionsfähigkeit dieses Finanzierungsprinzips wird gestört, wenn Personen mit hohem Einkommen und Gesunde sich diesen Umverteilungsmechanismen entziehen können (Sachverständigenrat 2008).

Ein zweiter zentraler Unterschied zwischen PKV und GKV besteht in unterschiedlichen Vergütungsstrukturen. Dies gilt insbesondere - aber nicht nur - für die ambulante ärztliche Versorgung (Walendzik et al. 2008). Der Arzt bekommt für gesetzlich versicherte Personen einen Mix aus Fallpauschalen und Einzelleistungsvergütungen bis zu einer Mengenobergrenze in Form des sogenannten Regelleistungsvolumens. Der gleiche Arzt erhält für privat versicherte Personen Einzelleistungsvergütungen ohne Mengenobergrenzen. Die aus diesem Nebeneinander von Vergütungssys- temen resultierenden finanziellen Anreize sind eindeutig. Privat versicherte Patienten sind deutlich attraktiver und werden bevorzugt behandelt. GKV-Versicherte führen im Vergleich zu PKV-Versicherten kürzere Gespräche mit ihrem Arzt, fühlen sich schlechter beraten und weniger an Entscheidungsprozessen beteiligt (Mielck/Helmert 2006b). Besserer Zugang in Form von kürzeren Wartezeiten für privat versicherte Personen ist inzwischen in der Empirie hinreichend robust dokumentiert (Lüngen et al. 2008). Diese Unterschiede im Zugang führen - im Gegensatz zu den Aussagen mancher ärztlichen Standesorganisationen - nicht nur zu Unterschieden in Service und Komfort. Es besteht schlichtweg die massive Gefahr von Unter- und Fehlversorgung, durch die vermeidbare gesundheitliche Schäden entstehen. Gleichzeitig besteht die Gefahr der Überversorgung von privat versicherten Patienten. Die Unterschiede im Vergütungssystem führen übrigens nicht nur zur Differenzierung der Versorgung in Abhängigkeit vom Versicherungsstatus. Sie führen auch zu einem starken Ausgabenwachstum in der PKV - eine der zentralen Ursachen für den ungebrochenen Prämienanstieg in der privaten Krankenversicherung (Grabka 2006; Reiners 2009).

Der Gesetzgeber hat die aus dem verzerrten Wettbewerb der beiden Kranken- versicherungssysteme resultierenden Probleme in der letzten Gesundheitsreform, dem GKV-Wettbewerbsstärkungsgesetz (GKV-WSG), im Jahr 2007 an zwei Stellen bearbeitet. Erstens wurde Arbeitnehmern mit einem Einkommen oberhalb der Versicherungspflichtgrenze der Wechsel in die private Krankenvollversicherung erschwert. Zweitens hat der Gesetzgeber zum 1. April 2007 den modifizierten Standardtarif sowie zum 1. Januar 2009 den Basistarif eingeführt. Nach der neuen Regelung müssen Arbeitnehmer in drei aufeinander folgenden Jahren ein Einkommen oberhalb der Versicherungspflichtgrenze erzielen, um in die private Krankenvollversicherung wechseln zu können. Diese Maßnahme wurde von der privaten Krankenversicherungsindustrie und jüngst auch von der FDP als Beeinträchtigung der Wahlfreiheit des betroffenen Personenkreises kritisiert (FDP 2009). Diese Interpretation ist jedoch nur teilweise zutreffend. Die Einschränkung der Wahlfreiheit dient dem Schutz der Versichertengemeinschaft in der GKV gegen die oben beschriebene Risikoselektion zugunsten der privaten Krankenversicherung. Eine Ausweitung der Wahlfreiheit, wie ebenfalls von der FDP gefordert, würde die negative Auslese zulasten der gesetzlichen Krankenversicherung noch weiter verstärken.

Die kurzfristige Wirksamkeit dieser Maßnahme ist eindeutig. Schon im Jahr 2007 hat sich der Nettoneuzugang in der PKV von 116.100 Personen auf 59.900 Versicherte fast halbiert. Auch im Jahr 2008 war der Nettoneuzugang mit 48.900 Personen deutlich geringer als zuvor. $\mathrm{Zu}$ diesem Personenkreis zählten 18.900 vorher nicht versicherte Personen (Verband der privaten Krankenversicherung 2008, 2009). Hält die Austrocknung des Neugeschäfts an, könnte es zu nicht einkalkulierten Prämiensteigerungen für Bestandsversicherte kommen. Das gilt vor allem für private Krankenversicherungsunternehmen, die ein differenziertes Tarifangebot mit Locktarifen für Neuversicherte vorhalten und damit auf einen stetigen Zufluss von günstigen Risiken aus der GKV angewiesen sind.

\footnotetext{
2 Pfaff et al. (2006, S. 178-182) errechneten für das Jahr 2003 einen Spielraum für Beitragssatzsenkungen von 1,1 Prozentpunkten durch die sofortige Einbeziehung der privat versicherten Angestellten, Selbstständigen und Beamten (einschließlich ihrer Familienangehörigen) in den GKV-Versichertenkreis.
} 
Die Einführung einer Versicherungspflicht auch für den der privaten Krankenversicherung zuzurechnenden Personenkreis (Beamte, Selbstständige, Arbeitnehmer mit einem Einkommen oberhalb der Versicherungspflichtgrenze) zum 1. Januar 2009 wurde durch die Einführung des Basistarifs begleitet. Das Leistungsangebot im Basistarif orientiert sich am Leistungskatalog der gesetzlichen Krankenversicherung, die Prämie darf den Höchstbeitrag in der GKV nicht übersteigen. Risikozuschläge dürfen im Basistarif nicht erhoben werden. Defizite im Basistarif müssen durch die Versicherten in den Normaltarifen finanziert werden. Der Basistarif führt demzufolge Elemente der Risikosolidarität in die private Krankenversicherung ein. Insbesondere diese „GKVisierung“ der $\mathrm{PKV}^{3}$ hat die private Krankenversicherungsindustrie dazu bewogen, vor dem Bundesverfassungsgericht gegen die beschriebenen Regelungen im GKV-WSG Verfassungsbeschwerde einzulegen. (Die Klage wurde mittlerweile abschlägig entschieden).

Zwar hat der Gesetzgeber durch den Kontrahierungszwang, den Ausschluss von Risikozuschlägen und den Höchstbeitrag im modifizierten Standardtarif die $\mathrm{Zu}$ gangsbarrieren gesenkt. Auch ist eine Kündigung eines privaten Krankenversicherungsvertrages nicht mehr möglich, wenn der Versicherte keinen anderweitigen Krankenversicherungsschutz nachweist. Gleichzeitig sind die zu zahlenden Prämien allerdings immer noch relativ hoch. Insbesondere für die zentrale Risikogruppe der Nichtversicherten, die sogenannten kleinen Selbstständigen ohne vormaligen gesetzlichen Krankenversicherungsschutz, stellt die Prämienhöhe damit weiterhin eine substanzielle Barriere dar (Greß et al. 2009).

Bisher werden der Basistarif und dessen Vorgänger, der modifizierte Standardtarif, vor allem von vormals nicht versicherten Personen nachgefragt. Dabei handelt es sich hauptsächlich um Personen mit Vorerkrankungen, die im Basistarif bezahlbaren Versicherungsschutz nachfragen können. Der PKV zufolge sind die entstehenden Leistungsausgaben im Basistarif etwa viermal so hoch wie die Prämieneinnahmen. ${ }^{4}$ Dies führt zu einem branchenweiten Prämienaufschlag von etwas mehr als $1 \%$ für die Versicherten außerhalb des Basistarifs.

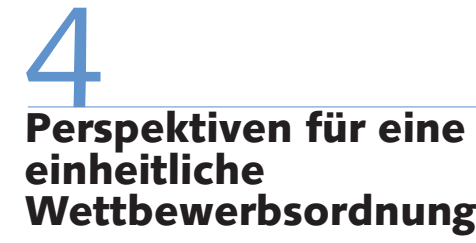

Durch das Wechselmoratorium hat der Gesetzgeber den Zufluss von guten Risiken in die private Krankenvollversicherung erschwert und gleichzeitig den Zugang für schlechte Risiken durch den Basistarif erleichtert. Von einem fairen Wettbewerb zwischen den Krankenversicherungssystemen kann jedoch noch immer keine Rede sein: Das System der gesetzlichen Krankenversicherung wird weiter durch Risikoselektion zugunsten der privaten Krankenversicherung belastet, und die finanziellen Anreize für die bevorzugte Behandlung privat versicherter Personen bleiben bestehen. Dabei hat die Versicherungspflichtgrenze als Trennwall zwischen schutzbedürftigem und nicht schutzbedürftigem Personenkreis ihre Rechtfertigung längst verloren, was auch der Sachverständigenrat zur Begutachtung der gesamtwirtschaftlichen Entwicklung in seinem Jahresgutachten 2008/2009 erkannt hat:

„Eine Wahlmöglichkeit nach Maßgabe der Erwerbsform, sich in einer privaten oder gesetzlichen Versicherung zu versichern, lässt sich mit diesem Argument einer Schutzbedürftigkeit nicht begründen. Darüber hinaus scheint sich seit der Etablierung privater Versicherungen die Funktion der Pflichtversicherungsgrenze darauf reduziert zu haben, den privaten Versicherungen ein Marktsegment zu sichern und somit Verkrustungen bei historisch gewachsenen Strukturen im Gesundheitswesen nicht aufbrechen zu müssen" (Sachverständigenrat 2008, S. 396).

Die Dualität von sozialer Krankenversicherung auf der einen Seite und substitutivem privaten Krankenversicherungsschutz auf der anderen Seite verliert somit, abgesehen vom Bestandsschutz der privaten Krankenversicherungsindustrie, ihre Rechtfertigung. Für einen fairen Wettbewerb zwischen gesetzlicher und privater Krankenversicherung ist eine einheitliche Wettbewerbsordnung eine unabdingbare Voraussetzung. Zentrale Rahmenbedingungen eines solchen Krankenversicherungssystems wären neben der allgemeinen Versicherungspflicht für die Bevölkerung der Kontrahierungszwang für die Krankenversicherer sowie ein einheitliches Ho- norierungssystem für die Leistungsanbieter. Denn ohne diese Rahmenbedingungen bestünde die Gefahr eines (Wieder-)Anwachsens der Zahl der Nichtversicherten, etwa wenn sich auf der Basis einer freiwilligen Versicherung die Bürger in ökonomisch schwierigen Zeiten gegen eine Krankenversicherung entscheiden oder Versicherte mit schlechten Risiken ohne Kontrahierungszwang keinen (bezahlbaren) Versicherungsschutz finden. Die wettbewerbsverzerrende Wirkung des dualen Honorierungssystems wurde bereits in Abschnitt 3 ausgeführt.

Wichtige Gestaltungsoptionen für eine einheitliche Wettbewerbsordnung liegen in der Beantwortung der folgenden Fragen:

(1) Soll überhaupt ein Weiterangebot von Krankenvollversicherungen durch private Krankenversicherungsunternehmen stattfinden?

(2) Falls ja: Soll die Krankenversicherung in der einheitlichen Wettbewerbsordnung rein privater Trägerschaft unterliegen oder im Wettbewerb zwischen privaten und gesetzlichen Anbietern organisiert werden?

(3) Soll das einheitliche System mit risikoäquivalenten Prämien ausgestaltet werden?

(4) Kann und soll eine einheitliche Wettbewerbsordnung Elemente der Kapitaldeckung enthalten?

$\mathrm{Zu}$ (1): Eine mögliche Option, das duale System aufzuheben, besteht darin, die Privatversicherung auf den komplementären Versicherungsschutz zu begrenzen, wie dies etwa in Österreich (Leiber 2006; Obinger/ Tálos 2006) und zahlreichen anderen Ländern (siehe Abschnitt 2) der Fall ist. Dies wäre auf Dauer technisch voraussichtlich

3 Parallel wurde durch die Einführung von neuen Wahltarifen eine "PKVisierung“ der GKV vorgenommen. Selbstbehalt-, Kostenerstattungstarife und Tarife für die Nichtinanspruchnahme von Leistungen sind vor allem für Versicherte mit guten Risiken attraktiv. Deren Einführung wurde damit begründet, eine Abwanderung guter Risiken in die PKV zu verhindern. Statt die ungleichen Wettbewerbsbedingungen zwischen PKV und GKV abzuschaffen, hat der Gesetzgeber jedoch neue Ungleichheiten innerhalb der Gruppe der GKV-Versicherten geschaffen (Leiber 2007).

4 So das Vorstandsmitglied der Debeka Versicherungsgruppe, Roland Weber, in einem Redebeitrag der Euroforum-Konferenz „PKV und GKV im Aufbruch“ am 25. Februar 2009 in Düsseldorf. 
die am wenigsten komplexe Lösung. Die heutige Versicherungspflichtgrenze könnte abgeschafft und alle Neuversicherten (einschließlich der Beamten und Selbstständigen) könnten in die Versicherungspflicht der GKV einbezogen werden. Für die Bestandsversicherten in der PKV müsste allerdings eine Übergangslösung gefunden werden, die deren erworbene Ansprüche schützt und zugleich die eingangs angesprochenen Defizite des dualen Systems abfedert. Ein gegebenenfalls auf einen bestimmten Zeitraum begrenztes Wahlrecht für die Bestandsversicherten, freiwillig in die GKV zu wechseln oder im PKV-Tarif zu bleiben, birgt die Gefahr, dass vor allem Versicherte mit schlechten Risiken vom Wechsel in die GKV Gebrauch machen. Dann müsste die PKV in den Risikostrukturausgleich integriert werden. Gleichzeitig ist diese Lösung politisch aufgrund des auf längere Sicht kompletten Wegfalls eines zentralen Geschäftsfeldes für die private Krankenversicherung wenig realistisch. Das würde insbesondere diejenigen Unternehmen hart treffen, die in der Vergangenheit wenig in den Markt für komplementären Versicherungsschutz investiert haben und keine anderen Versicherungssparten betreiben.

$\mathrm{Zu}$ (2): Falls der Gesetzgeber sich dagegen entscheidet, das Vollversicherungsangebot durch die PKV aufzugeben, um das Geschäftsfeld dieser Unternehmen zu schützen, bestünde eine weitere Möglichkeit zur Schaffung einer einheitlichen Wettbewerbsordnung darin, wie in den Niederlanden geschehen und jüngst auch von der FDP gefordert, die gesetzlichen Krankenkassen zu privatisieren. In den Niederlanden hatte diese Maßnahme den Sinn, die Zustimmung der privaten Krankenversicherer und der Arbeitgeber zu der Reform sicherzustellen. Für die Funktionalität des einheitlichen Krankenversicherungsmarktes ist eine Privatisierung der öffentlichrechtlichen Krankenkassen jedoch weder hinreichend noch notwendig. Die Privatisierung birgt vor dem Hintergrund europarechtlicher Regelungen sogar eine Reihe von Gefahren. Es ist unklar, ob der Europäische Gerichtshof (EuGH) bei einer entsprechenden Klage eines in der EU niedergelassenen Krankenversicherers die umfassende nationale Regulierung privater Krankenversicherungsunternehmen akzeptieren würde (Paolucci et al. 2006). ${ }^{5}$

Zudem können sich formal private Modelle in ihrer rechtlichen Ausgestaltung deutlich unterscheiden. In den Niederlanden ist die private Krankenversicherung häufig in Form von Versicherungsvereinen auf Gegenseitigkeit $(\mathrm{VVaG})^{6}$ organisiert und unterliegt einer umfassenden staatlichen Rahmenregulierung - einschließlich einer Versicherungspflicht für die Bevölkerung, des Kontrahierungszwangs für die Krankenversicherer, eines Verzichts auf Kapitaldeckung sowie des Verzichts auf risikoäquivalente Prämien.

$\mathrm{Zu}$ (3): In Deutschland wird immer wieder, zuletzt im Konzept der sogenannten Bürgerprivatversicherung (zuletzt Eekhoff et al. 2009), die Forderung nach einer umfassenden, privaten Krankenvollversicherung mit risikoäquivalenten Prämien erhoben. Erstens sei der Versuch, durch den Risikostrukturausgleich Anreize zur Selektion zu neutralisieren, zum Scheitern verurteilt. Zweitens werde durch risikoäquivalente Prämien das Verhältnis von Prämie und Leistung für die Versicherten besser erkennbar (ebd.). Diese Forderung verkennt jedoch, dass risikoäquivalente Prämien für Menschen mit chronischen Erkrankungen schnell unbezahlbar werden. Um die Prämien für die Betroffenen finanzierbar zu machen, wird wieder ein Ausgleichmechanismus notwendig, der auf der Grundlage des gesundheitlichen Risikos Kompensationszahlungen für die Betroffenen zur Verfügung stellt. Damit sind jedoch die vorgeblichen Vorteile einer einheitlichen Wettbewerbsordnung mit risikoäquivalenten Prämien - Verzicht auf einen Risikostrukturausgleich und transparenteres Verhältnis von Prämie und Leistung - hinfällig.

$\mathrm{Zu}$ (4): Wenn der Gesetzgeber ein rein privatwirtschaftlich organisiertes System umgehen möchte, wäre eine einheitliche Wettbewerbsordnung auch im freien Wettbewerb öffentlich-rechtlicher Krankenkassen und privater Krankenversicherungsunternehmen um alle Versicherten gestaltbar. Dies müsste allerdings auf der Basis eines standardisierten Leistungspaketes und unter Verzicht auf risikoäquivalente Prämien geschehen. Jüngere Studien haben gezeigt, dass sich eine solche Wettbewerbsordnung unabhängig von der Entscheidung über die Frage installieren lässt, ob die privaten Krankenversicherer weiterhin im Kapitaldeckungsverfahren oder im Umlageverfahren finanziert werden (Albrecht et al. 2006). Auch Mischmodelle sind denkbar. Das Umlageverfahren hat den Vorteil, dass sich Krankenversicherer nicht in die Abhängig- keit von Kapitalmärkten begeben müssen. Zwar haben die privaten Krankenversicherer auch im Jahr 2008 wegen der vergleichsweise rigorosen Vorschriften für die Anlage der Alterungsrückstellungen noch positive Renditen erwirtschaftet. Kommt es jedoch durch die Finanzkrise zu Totalausfällen bei Unternehmens- oder sogar bei Staatsanleihen, ist sogar mit negativen Renditen zu rechnen.

Die Vereinbarkeit einer einheitlichen Wettbewerbsordnung mit Kapitaldeckung ist kein grundsätzliches, sondern ein technisches Problem. In der Vergangenheit hat die private Versicherungsindustrie die Individualisierung und Mitgabe von Alterungsrückstellungen als nicht machbar gebrandmarkt (PKV-Publik 2006). Inzwischen sind jedoch immer mehr Stimmen zu hören, die eine grundsätzliche Machbarkeit sowie Vereinbarkeit mit den Grundrechten der privat Versicherten und Versicherungsunternehmen (Bieback 2006) nicht infrage stellen und Modelle zur Umsetzung entwickeln (z. B. Sehlen et al. 2006).

Ein solches Modell wäre auch mit dem neu eingeführten Gesundheitsfonds kompatibel. Dadurch, dass die Beiträge der Versicherten nun bereits von den Zahlungen an ihre Krankenkasse abgekoppelt sind, lassen sich die Mitglieder der PKV leicht in das einkommensabhängige Beitragssystem und Umverteilungssystem einbeziehen. Die Mitglieder der privaten Versicherungen könnten über einkommensabhängige Beitragszahlungen ebenfalls in den Fonds einbezogen werden. Auch die privaten Versicherungen würden dann wie die gesetzlichen für ihre Versicherten jeweils einen ri-

5 Im Fall der niederländischen Reform hat sich die dortige Regierung zwar von der Europäischen Kommission die europarechtliche Kompatibilität des Modells bestätigen lassen. Unklar ist jedoch, was passiert, falls es einmal zu einer Klage vor dem EuGH kommen sollte, insbesondere wenn ausländische Anbieter am niederländischen Versicherungsmarkt partizipieren wollen.

6 Da ein VVaG keine fremden Eigentümer hat, die Ansprüche auf den erzielten Gewinn haben, verbleiben erwirtschaftete Überschüsse im Unternehmen oder sie kommen den Versicherungsnehmern als Vereinsmitgliedern zu. Allerdings kann ein VVaG nicht ohne weiteres Kapital auf dem Kapitalmarkt aufnehmen, die dafür benötigten Sicherheitsmittel müssen aus seinen Gewinnen selbst erbracht werden. Dies schränkt die Möglichkeiten ein, Gewinne zugunsten der Versicherungsnehmer zu verwenden. Darüber hinaus kann ein $\mathrm{VVaG}$ seine Geschäftstätigkeit nicht so leicht ausweiten, da er die dafür benötigten Sicherheitsmittel erst aus dem bestehenden Geschäft erwirtschaften muss. 
sikoadjustierten Pauschalbeitrag aus dem Fonds erhalten. Prämieanteile für über den GKV-Katalog hinausgehende Leistungen müssten von den privat Versicherten selbst getragen werden (Leiber 2009).

Ein wesentliches Merkmal einer einheitlichen Wettbewerbsordnung wäre, dass die private Krankenversicherungsindustrie den substitutiven privaten Krankenversicherungsschutz in seiner heutigen Form als Markt verlieren würde. Die Existenzberechtigung privater Krankenversicherer würde jedoch nicht infrage gestellt werden, da sich gleichzeitig das Geschäftsfeld der PKV im Wettbewerb mit der GKV um die GKV-äquivalenten Standardversicherungen erweitern würde. Daneben könnten Zusatzversicherungen für darüber hinausgehende Leistungen angeboten werden.

Eine einheitliche Wettbewerbsordnung würde einen weitreichenden Reformschritt darstellen, für den die politischen Konsenserfordernisse nicht einfach zu erzielen sind. Bis zur Entscheidung über eine einheitliche Wettbewerbsordnung könnten Probleme des Nebeneinanders zwischen gesetzlicher Krankenversicherung und substitutiver privater Krankenversicherung durch zwei Maßnahmen wesentlich abgemildert werden. Erstens könnte ein Einbezug der privat krankenversicherten Personen in den Risikostrukturausgleich der GKV die finanziellen Konsequenzen der Risikoselektion zugunsten der privaten Krankenversicherung kompensieren. Konzeptionelle Vorarbeiten zur Umsetzung dieser Maßnahme liegen vor und haben die Durchführbarkeit bestätigt (Albrecht et al. 2006; Greß/Wasem 2004).

Schwieriger gestaltet sich zweitens die Neutralisierung der finanziellen Anreize zur bevorzugten Behandlung des privat versicherten Personenkreises. Eine für die Ärzteschaft insgesamt aufkommensneutrale Angleichung der Vergütungssysteme würde die GKV belasten und gleichzeitig PKV und Beihilfeträger entlasten. Nach Berechnungen aus dem Jahr 2008, bevor zu Jahresbeginn 2009 das Honorarsystem eingeführt wurde, würde die GKV durch die Vergütungsangleichung um rund 3,2 Mrd. $€$ belastet werden (Walendzik et al. 2008). Beide Maßnahmen, die Ausweitung des Risikostrukturausgleichs auf die PKV und die Neutralisierung der finanziellen Anreize zur bevorzugten Behandlung des privat versicherten Personenkreises, sind daher als Bündel zu betrachten. Sie stellen darüber hinaus - und hier ist wiederum auf die Erfahrungen in den Niederlanden zu verweisen - entscheidende Vorarbeiten für die Einführung einer einheitlichen Wettbewerbsordnung im System der deutschen Krankenversicherung dar. Unter anderem könnten Widerstände aus der Ärzteschaft gegen eine Modifikation der PKV auf diese Weise aufgefangen werden.

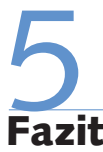

Dieser Beitrag zeigt, dass die deutsche, in den entwickelten Industrieländern fast einzigartige Dualität von privater und gesetzlicher Krankenversicherung mit einer Reihe gravierender Probleme verbunden ist. Auch die Gesundheitsreform von 2007 konnte diese Probleme nur begrenzt lösen. Im wissenschaftlichen Diskurs herrschte bereits vor der Bundestagswahl 2005 weitgehende Einigkeit über die Defizite des dualen Systems. Eine Überwindung dieser Trennung fand sowohl bei den Befürwortern einer einkommensabhängigen Bürgerversicherung Zustimmung als auch bei einigen Anhängern einkommensunabhängiger Pauschalprämien. Allerdings schreckte ausgerechnet an dieser Stelle, an der in der Wissenschaft Konsens bestand, der Gesetzgeber vor Veränderungen zurück.

Auch vor der Bundestagswahl 2009 besteht also weiterhin die Notwenigkeit zur Herstellung einer einheitlichen Wettbewerbsordnung. Um diese zu erreichen, steht dem Gesetzgeber prinzipiell eine Reihe von Handlungsmöglichkeiten mit jeweils unterschiedlichen technischen und politischen Implikationen zur Verfügung. Eine Privatisierung der Trägerschaft nach niederländischem Vorbild könnte politische Widerstände in den Reihen von Arbeitgebern und der PKV abmildern, wäre jedoch mit sehr hohen Anforderungen an die staatliche Regulierung der Versicherungsbedingungen sowie mit dem Risiko europarechtlicher Eingriffe in die Gestaltung des Gesundheitssystems verbunden.

Eine Umstellung eines solchen Systems gar auf risikoäquivalent kalkulierte Prämien würde dazu führen, dass Menschen mit chronischen Erkrankungen nur schwer einen (bezahlbaren) Versicherungsschutz finden. Dies könnte nur durch einen sozialen Ausgleichmechanismus verhindert werden, der die vorgeblichen Vorteile risikoäquivalenter Prämien - Verzicht auf einen Risikostrukturausgleich und transparenteres Verhältnis von Prämie und Leistung - wieder aufhebt.

Eine Begrenzung der PKV auf den komplementären Versicherungsschutz wäre nach Auslaufen der PKV-Bestandsversicherungen voraussichtlich die technisch am wenigsten komplexe Lösung. Diese hätte jedoch mit hohen politischen Hürden zu kämpfen und würde sich auf PKV-Unternehmen negativ auswirken, die sich bislang vorwiegend auf den Vollversicherungsmarkt konzentrieren.

Eine Wettbewerbsordnung, in der gesetzliche und private Versicherungen unter einheitlichen Rahmenbedingungen konkurrieren, ist, anders als lange Zeit von der privaten Versicherungswirtschaft behauptet, zwar technisch komplex, jedoch lösbar, mit Kapitaldeckung vereinbar und stellt die Existenzberechtigung der PKV nicht infrage.

Bis eine vollständige Aufhebung der Dualität verwirklicht ist, wären der Einbezug des derzeit privat versicherten Personenkreises in den Risikostrukturausgleich der GKV und eine für die Ärzteschaft einkommensneutrale Angleichung der Vergütungssysteme wichtige erste Schritte zur Überwindung der angesprochenen Defizite des dualen Systems. 


\section{LITERATUR}

Albrecht, M./Hofmann, J./Reschke, P./Schiffhorst, G./Sehlen, S. (2006) Stabilisierung der Finanzierungsbasis und umfassender Wettbewerb in einem integrierten Krankenversicherungssystem, Berlin

Bieback, K.-J. (2006): Einbeziehung der Altersrückstellungen der PKV in die erweiterte GKV, in: Albrecht, M./Schräder, W. F./Sehlen, S. (Hrsg.): Modelle einer integrierten Krankenversicherung: Finanzierungseffekte, Verteilungswirkungen, Umsetzung, Berlin, S. 143-206

Carey, D./Herring, B./Lenain, P. (2009): Health Care Reform in the United States, OECD Economics Department Working Papers, No. 665, doi: 10.1787/227104360465, Paris

Colombo, F./Tapay, N. (2004): Private Health Insurance in OECD Countries: The Benefits and Costs for Individuals and Health Systems, OECD Health Working Paper No. 15, Paris

Eekhoff, J./Bünnagel, V./Kochskämper, S./Menzel, K. (2009): Die Bürgerprivatversicherung: Nachhaltigkeit und Effizienz für das deutsche Gesundheitssystem, Köln

FDP (2009): Für ein einfaches, transparentes und leistungsgerechtes Gesundheitswesen, Entschließungsantrag der FDP-Bundestagsfraktion, Drucksache 16/11879 vom 11.02., Berlin

Grabka, M. M. (2006): Prämien in der PKV: Deutlich stärkerer Anstieg als in der gesetzlichen Krankenversicherung, in: DIW-Wochenbericht 46, S. 653-659

Greß, S. (2005): The Role of Private Health Insurance in Social Health Insurance Countries - Implications for Canada, in: Flood, C./Roach, K./ Sossin, L. (Hrsg.): Access to Care - Access to Justice. The Legal Debate Over Private Health Insurance in Canada, Toronto, S. 278-295

Greß, S. (2007): Private Health Insurance in Germany: Consequences of a Dual System, in: Healthcare Policy 2, S. 29-37

Greß, S./Manouguian, M./Wasem, J. (2007): Niederlande: Anatomie einer Reform, in: Gesundheit und Gesellschaft 1, S. 36-40

Greß, S./Walendzik, A./Wasem, J. (2009): Auswirkungen der Maßnahmen gegen Nichtversicherung im GKV-WSG - Eine Zwischenbilanz, Sozialer Fortschritt/German Review of Social Policy, im Erscheinen Greß, S./Wasem, J. (2003): Private Krankenversicherung in der Europäischen Union - Auswirkungen institutioneller Variationen am Beispiel Deutschlands und der Niederlande, in: Cassel, D. (Hrsg.): Europäische Gesundheitssysteme: Gestaltungsprobleme und Lösungsansätze, BadenBaden, S. 21-33

Greß, S./Wasem, J. (2004): Integration der Privaten Krankenversicherung (PKV) in den Risikostrukturausgleich der Gesetzlichen Krankenversicherung (GKV) - Eine funktional äquivalente Alternative zur Bürgerversicherung?, in: Recht und Politik im Gesundheitswesen 2, S. 37-44 Leiber, S. (2006): Wohin steuert die Bismarcksche Sozialversicherung: Aktuelle Gesundheitsreformen im Vergleich, in: Schäfer, C./Seifert, H. (Hrsg.): Kein bisschen leise: 60 Jahre WSI, Hamburg, S. 57-74 Leiber, S. (2007): Nach der Reform ist vor der Reform: Ungelöste Fragen der Gesundheitsreform 2007, in: WSI-Mitteilungen 9, S. 508-510 Leiber, S. (2009): Die Stärken nutzen: Vorschläge zur Weiterentwicklung des Gesundheitsfonds, in: WSI-Mitteilungen 3, S. 168-170

Leiber, S./Manouguian, M. (2009): Vereinbarkeit von Wettbewerb und Solidarität in der sozialen Krankenversicherung? Gesundheitsreformen in den Niederlanden und Deutschland, in: Böckmann, R. (Hrsg.): Gesundheitsversorgung zwischen Wettbewerb und Solidarität, Wiesbaden, S. $175-202$
Lüngen, M./Stollenwerk, B./Messner, P./Lauterbach, K. W./Gerber, A. (2008): Waiting times for elective treatments according to insurance status: A randomized empirical study in Germany, in: International Journal for Equity in Health 1, S. doi: 10.1186/1475-9276-1187-1181 Mielck, A./Helmert, U. (2006a): Vergleich zwischen GKV- und PKV-Versicherten: Unterschiede bei Morbidität und gesundheitlicher Versorgung, in: Böcken, J./Braun, B./Amhof, R./Schnee, M. (Hrsg.): Gesundheitsmonitor 2006, Gütersloh, S. 32-52

Mielck, A./Helmert, U. (2006b): Das Arzt-Patienten-Verhältnis in der ambulanten Versorgung: Unterschiede zwischen GKV- und PKV-Versicherten, in: Böcken, J./Braun, B./Amhof, R./Schnee, M. (Hrsg.): Gesundheitsmonitor 2006, Gütersloh, S. 114-132

Mossialos, E./Thomson, S. (2004): Voluntary health insurance in the European Union: World Health Organization, Brussels

Obinger, H./Tálos, E. (2006): Die Krankenversicherung in Österreich Ein Erfolgsmodell?, in: WSI-Mitteilungen 4, S. 220-226

Paolucci, F./Exter, A. D./Van De Ven, W. (2006): Solidarity in competitive health insurance markets: analysing the relevant $\mathrm{EC}$ legal framework. in: Health Economics, Policy and Law 2, S. 107-126

Pfaff, A. B./Langer, B./Mamberer, F./Pfaff, M./Freund, F./Holl, N. (2006): Finanzierungsalternativen der Gesetzlichen Krankenversicherung: Einflussfaktoren und Optionen zur Weiterentwicklung, Berlin PKV-Publik (2006): Portabilität: Theoretisch untauglich, praktisch unmöglich. Informationen des Verbands der privaten Krankenversicherungen e. V., in: PKV-Publik 6, S. 63-65

Reiners, H. (2009): Mythen der Gesundheitspolitik, Bern

Rosenau, P. V./Lako, C. J. (2008): An Experiment with Regulated Competition and Individual Mandates for Universal Health Care: The New Dutch Health Insurance System, in: Journal of Health Politics, Policy and Law 6, S. 1031-1055

Sachverständigenrat zur Begutachtung der gesamtwirtschaftlichen Entwicklung (2008): Die Finanzkrise meistern - Wachstumskräfte stärken, Jahresgutachten 2008/2009, Wiesbaden

Sehlen, S./Hofmann, J./Reschke, P. (2006): Möglichkeiten zur Einbeziehung von gesetzlich und privat Krankenversicherten in eine integrierte Krankenversicherung, in: Albrecht, M./Schräder, W. F./Sehlen, S. (Hrsg.): Modelle einer integrierten Krankenversicherung: Finanzierungseffekte, Verteilungswirkungen, Umsetzung, Berlin, S. 89-142

Verband der Privaten Krankenversicherung (2008): Die private Krankenversicherung - Zahlenbericht 2007/2008, Köln

Verband der Privaten Krankenversicherung (2009): Politischer Nackenschlag der Gesundheitsreform auch 2008 beim Netto-Neuzugang zu spüren, Presserklärung des Verbandes der privaten Krankenversicherung vom 25. März, Köln/Berlin Walendzik, A./Greß, S./Manouguian, M./Wasem, J. (2008): Vergütungsunterschiede im ärztlichen Bereich zwischen PKV und GKV auf Basis des standardisierten Leistungsniveaus der GKV und Modelle der Vergütungsangleichung, Diskussionsbeitrag Nr. 165, Fachbereich Wirtschaftswissenschaften der Universität Duisburg-Essen, Campus Essen 